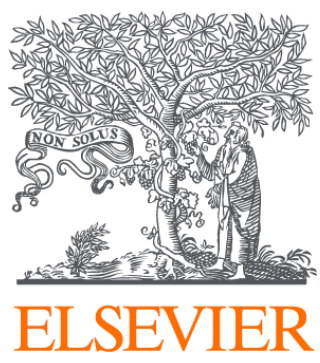

Since January 2020 Elsevier has created a COVID-19 resource centre with free information in English and Mandarin on the novel coronavirus COVID-

19. The COVID-19 resource centre is hosted on Elsevier Connect, the company's public news and information website.

Elsevier hereby grants permission to make all its COVID-19-related research that is available on the COVID-19 resource centre - including this research content - immediately available in PubMed Central and other publicly funded repositories, such as the WHO COVID database with rights for unrestricted research re-use and analyses in any form or by any means with acknowledgement of the original source. These permissions are granted for free by Elsevier for as long as the COVID-19 resource centre remains active. 


\title{
Excess mortality during the COVID-19 pandemic: a geospatial and statistical analysis in Mogadishu, Somalia
}

\author{
Abdihamid Warsame $^{1, *}$, Farah Bashiir ${ }^{2}$, Terri Freemantle ${ }^{3}$, Chris Williams ${ }^{3}$, \\ Yolanda Vazquez ${ }^{3}$, Chris Reeve ${ }^{3}$, Ahmed Aweis ${ }^{2}$, Mohamed Ahmed ${ }^{2}$, Francesco Checchi ${ }^{1}$, \\ Abdirisak Dalmar ${ }^{2}$ \\ ${ }^{1}$ Faculty of Epidemiology and Population Health, The London School of Hygiene and Tropical Medicine, London, United Kingdom \\ ${ }^{2}$ Somali Disaster Resilience Institute, Mogadishu, Somalia \\ ${ }^{3}$ Satellite Applications Catapult, Didcot, London, United Kingdom
}

\section{A R T I C L E I N F O}

\section{Article history:}

Received 4 June 2021

Revised 20 September 2021

Accepted 21 September 2021

\section{Keywords:}

mortality

COVID-19

humanitarian

epidemic

evaluation

\begin{abstract}
A B S T R A C T
Background: While the impact of the COVID-19 pandemic has been well documented in high-income countries, less is known about the health effects in Somalia, where health systems are weak and vital registration is underdeveloped.

Methods: We used remote sensing and geospatial analysis to quantify burial numbers from January 2017 to September 2020 in Mogadishu. We imputed missing grave counts using surface area data. Simple interpolation and a generalised additive mixed growth model were used to predict actual and counterfactual burial rates by cemetery and across Mogadishu during the most likely period of COVID-19 excess mortality and to compute excess burials. We undertook a qualitative survey of key informants to determine the drivers of COVID-19 excess mortality.

Results: Burial rates increased during the pandemic, averaging 1.5 -fold and peaking at a 2.2 -fold increase on pre-pandemic levels. When scaled to plausible range of baseline crude death rates, the excess death toll between January and September 2020 was 3200-11 800. Compared with Barakaat Cemetery Committee's burial records, our estimates were lower.

Conclusions: Our study indicates considerable underestimation of the health effects of COVID-19 in Banadir and an overburdened public health system struggling to deal with the increasing severity of the epidemic in 2020.
\end{abstract}

(c) 2021 The Author(s). Published by Elsevier Ltd on behalf of International Society for Infectious

Diseases.

This is an open access article under the CC BY-NC-ND license

(http://creativecommons.org/licenses/by-nc-nd/4.0/)

\section{Introduction}

As a result of three decades of protracted conflict and state fragility, Somalia's health system is considered weak and reports some of the worst health indicators in the world (Directorate of National Statistics Federal Government of Somalia, 2020). The emergence of the COVID-19 pandemic has exacerbated the effects of ongoing humanitarian crises caused by natural disasters and conflict (UNDP, 2020; UNSC, 2020).

As of 6 May 2021, the Somali government reported 14368 cumulative COVID-19 cases and 745 deaths due to COVID-19 (Ministry of Health and Human Services FG of S. COVID-19 Dash-

\footnotetext{
* Corresponding author.

E-mail address: Abdihamid.warsame@lshtm.ac.uk (A. Warsame).
}

board, Somalia 2020). Although these figures are much lower than those of countries with robust public health infrastructure, there is concern that they do not fully reflect the reality of the epidemic in the country. As a result of the country's very low COVID19 testing capacity, the potential stigma associated with COVID19 , and limited access to many parts of the country, the official data may not be an accurate representation of the true burden of COVID-19 (UNFPA Somalia, 2020; WHO, 2020). Investigative enquiries by news organizations and anecdotal reports point to larger numbers of COVID-19 deaths than have been officially reported (BBC News, 2020; Jason Burke and Abdalle Ahmed Mummin, 2020; Wariyaha Muqdisho, 2020). We attempted to shed light on the health effects of COVID-19 in Mogadishu by estimating excess mortality using satellite imagery analysis first developed for use in Yemen (Besson EK, Norris A, Bin Ghouth AS, Freemantle T, Alhaffar 


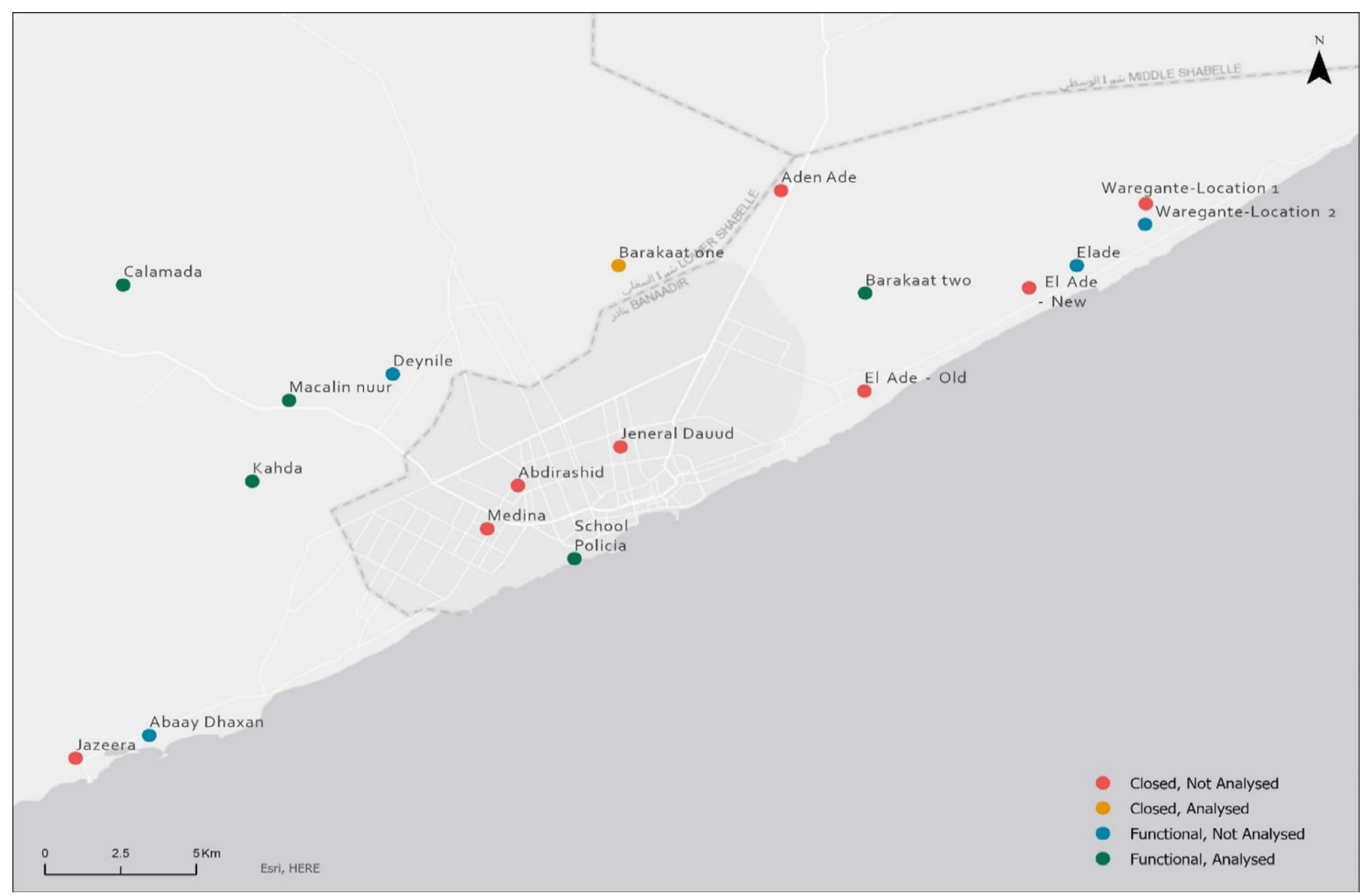

Figure 1. Location and status of cemeteries in Banadir September 2020

M, Vazquez Y, 2020). We also highlighted key contextual drivers using qualitative methods.

\section{Methods}

\section{Study population and period}

We estimated excess mortality attributable to COVID-19 and related social and health systems disruptions among people living in the Banadir region, which is almost entirely occupied by the rapidly expanding urban and peri-urban conurbation of Mogadishu. To estimate a baseline death rate, we defined a baseline period from 1 January 2016 to 31 December 2019, i.e., before widespread SARS-CoV-2 transmission in Mogadishu; our analysis period for the epidemic was 1 January to 16 September 2020. Based on the assumption that all decedents in Banadir are buried in recognised cemeteries, we sought to identify and collect data on every 'active' cemetery (i.e., receiving new burials) at any point during the analysis period.

\section{Data collection}

\section{Cemetery identification}

We identified an initial list of cemeteries in the Banadir region by analysing open-source location data and satellite imagery (Google Earth, 2021, Google Maps, 2021, OpenStreetMap, 2021) supplemented by the field observations of local researchers. Our analysis excluded five private or family-owned cemeteries - all five appeared active upon visitation, but we were constrained by vegetation cover for Abaay Dhaxan and the inability to procure suit- able satellite imagery for Jazeera and El Ade (Figure 1). A list of all cemeteries identified and visited is included in the supplementary file.

\section{Satellite imagery}

The analysis relied on the availability of suitable archive satellite imagery for the analysis period, with the required pixel resolution and minimum visual obstruction. We sourced the most suitable very high resolution (VHR) images available for each cemetery, ensuring they were cloud-free, and of high radiometric quality; we focused on images with a spatial resolution of $\sim 31-40 \mathrm{~cm}$ per pixel. We selected the higher resolution of $31 \mathrm{~cm}$ where available, as it provided the best clarity of features on the ground and ensured the most accurate identification of new burials. We used lower resolution imagery $(\sim 40-50 \mathrm{~cm})$ where no higher resolution was available, to investigate its uses, limitations and, at a minimum, measure surface area change. Where available, we obtained at least one satellite image per year for 2016-2019 and monthly imagery for January-October 2020. Images were purchased as Ortho Natural Colour via SecureWatch (Maxar, 2021) and were delivered pre-processed, corrected for illumination and geometric distortion, and pan-sharpened. To further improve the detection of individual burial plots, where beneficial, some were enhanced using Edge Detection techniques (Sobel and/or Touzi filter (Centre national d'études spatiales, 2019)).

\section{Other burial data sources}

We obtained monthly burial reports from the Barakaat Cemetery Development Committee covering April-June 2019 and January-April 2020. 
Table 1

Estimates (95\% confidence intervals) from retrospective sample household surveys conducted in Banadir Region during 2013-2018 by humanitarian actors. Surveys investigated a retrospective period of 87-104 days. CDR $=$ Crude death rate per 10,000 per day. Source: United Nations Food Security and Nutrition Analysis Unit for Somalia.

\begin{tabular}{llllll}
\hline livelihood & year & month & season & CDR & U5DR \\
\hline displaced & 2013 & November & Deyr & $0.62(0.3$ to 1.3$)$ & $0.5(0.16$ to 1.54$)$ \\
displaced & 2015 & May & Gu & $0.63(0.33$ to 1.23$)$ & $1.36(0.72$ to 2.54$)$ \\
urban & 2015 & May & Gu & $0.54(0.32$ to 0.92$)$ & $0.64(0.27$ to 1.49$)$ \\
urban & 2015 & November & Deyr & $0.28(0.15$ to 0.53$)$ & $0.23(0.06$ to 0.96$)$ \\
agropastoralists & 2016 & May & Gu & $0.35(0.16$ to 0.73$)$ & $1.02(0.38$ to 2.74$)$ \\
displaced & 2016 & November & Deyr & $0.61(0.34$ to 1.11$)$ & $0.74(0.3$ to 1.81$)$ \\
displaced & 2017 & May & NA & $0.7(0.36$ to 1.33$)$ & $1.28(0.53$ to 3.04$)$ \\
urban & 2017 & May & NA & $0.68(0.46$ to 1.01$)$ & $0.69(0.32$ to 1.49$)$ \\
displaced & 2018 & March & NA & $0.42(0.27$ to 0.65$)$ & $0.6(0.25$ to 1.41$)$ \\
displaced & 2018 & May & Gu & $1.06(0.73$ to 1.54$)$ & $2.56(1.54$ to 4.22$)$ \\
urban & 2018 & June & Gu & $0.25(0.11$ to 0.54$)$ & $0.34(0.08$ to 1.38$)$ \\
displaced & 2018 & November & Deyr & $0.74(0.48$ to 1.12$)$ & $1.21(0.58$ to 2.53$)$ \\
urban & 2018 & November & Deyr & $0.15(0.05$ to 0.47$)$ & $0.19(0.03$ to 1.46$)$ \\
\hline
\end{tabular}

Key informant interviews

A total of $20 \mathrm{key}$ informant interviews were conducted with health authorities and professionals, gravediggers, religious leaders, and other stakeholders to provide insights into community perception on COVID-19 mortality, community practice during the pandemic and challenges (Table S2).

\section{Analysis}

\section{Population denominators}

To calculate burial rates per population, we computed population denominators for Banadir Region using two starting sources: estimates by the WorldPop (Wordpop Gridded Population Estimate Datasets and Tools, 2020) projects for 2015 and 2020 (assuming December as the month in which the estimate was centred). WorldPop redistributes existing countrywide estimates (e.g., through census exercises) across space using a predictive statistical model of population density per $100 \mathrm{~m}^{2}$ pixels, built from various remotely-sensed variables, including vegetation index and settlement pattern. (Linard C, Gilbert M, W. Snow R, Abdisalan M. Noor, 2012). Notably, for Somalia, the WorldPop project has developed methods to account for settlements for displaced persons. We forward-calculated population from 2015 and back-calculated from 2020, by using a natural growth rate of 30 per 1000 per year (assumed crude birth rate of 44 per 1000 per year minus crude death rate (CDR) of 15 per 1000 per year [the latter an average of existing surveys from Banadir Region: see Table 1]) and by accounting for population movement in and out of the region over time, as reported by the United Nations High Commissioner for Refugees' Protection and Return Monitoring Project (UNHCR, n.d.). The two alternative base estimates yield 'high' and 'low' scenarios (Figure 2), which we carried into further estimation steps.

\section{Imagery analysis}

For the burial assessment, we combined manual and semiautomated image analysis approaches. The approaches applied were determined by the suitability of the input satellite imagery to a specified set of criteria, including image quality, image resolution, cloud cover, the typology of the cemetery (formal vs informal arrangement, infilling vs new blocks) and the ability to identify individual burial plots by either a spectral or object-based image processing technique.

A small number of sites where imagery was most consistent with the criteria (i.e., those in which the individual burial plots could be clearly observed) were analysed using a computer vision technique developed in Python to localise the georeferenced perimeter of regularly shaped, small, bright objects in optical satel- lite imagery. First, we applied a simple binary threshold to a single image band to identify 'bright' pixels in a region of interest. Then we applied an opening morphological transformation to remove isolated pixels, leaving larger 'blobs' of high reflection. We ignored 'blobs' exceeding a predefined pixel size as background clutter.

To delineate the individual burials, we utilised an active contour model algorithm (Chan and Vese, 2001). We converted features that satisfied size and shape constraints into real-world coordinates using image geo-transform coefficients and exported as polygon vector geometry. The output features were quality checked manually by an analyst. In most situations, subsequent imagery was not of high enough quality to meet the criteria required for the computer vision technique and features were only used as a reference dataset during the manual annotation. Any sites classified as not meeting the pre-defined criteria were assessed manually by trained annotators. An analyst can annotate a satellite image by assigning semantic labels that represent certain features as objects. Although more time consuming, manual annotation results in lower uncertainty and higher precision when compared with automated and semi-automated annotation techniques. All annotations were quality checked by a second analyst to ensure accuracy. The analysts were trained and qualified in remote sensing and GIS, with experience working with satellite imagery. The use of expert annotators to create training label data was undertaken because the extraction of burial information from satellite imagery is a new and relatively untested approach (Besson et al., 2020). Furthermore, accurately labelled data is a prerequisite for applying automated machine learning-based approaches that could facilitate similar analyses at scale in future.

As outlined above, for burial sites where individual burial plots were visible in the satellite imagery, object-based image annotation of features (Cloudfactory, 2020) was undertaken to ascertain the number of new burials. However, due to limited image resolution coupled with the degradation of grave visibility over time (resulting from local environmental and climate dynamics, such as erosion and sand migration), it was not always possible to annotate individual burial plots. In these circumstances, annotation was undertaken using a 'bounding box' approach allowing for the calculation of total burial area over time, this area, coupled with a known conversion factor, acted as the indicator of burial change.

\section{Statistical analysis}

Inferring missing grave counts. Overall, 68 sequential satellite images were available across the six analysed cemeteries (mean 11.2 per cemetery). However, some images were constrained by cloud cover or poor data quality (e.g., inadequate resolution), such that, for a given time point, individual graves were not visible for a frac- 


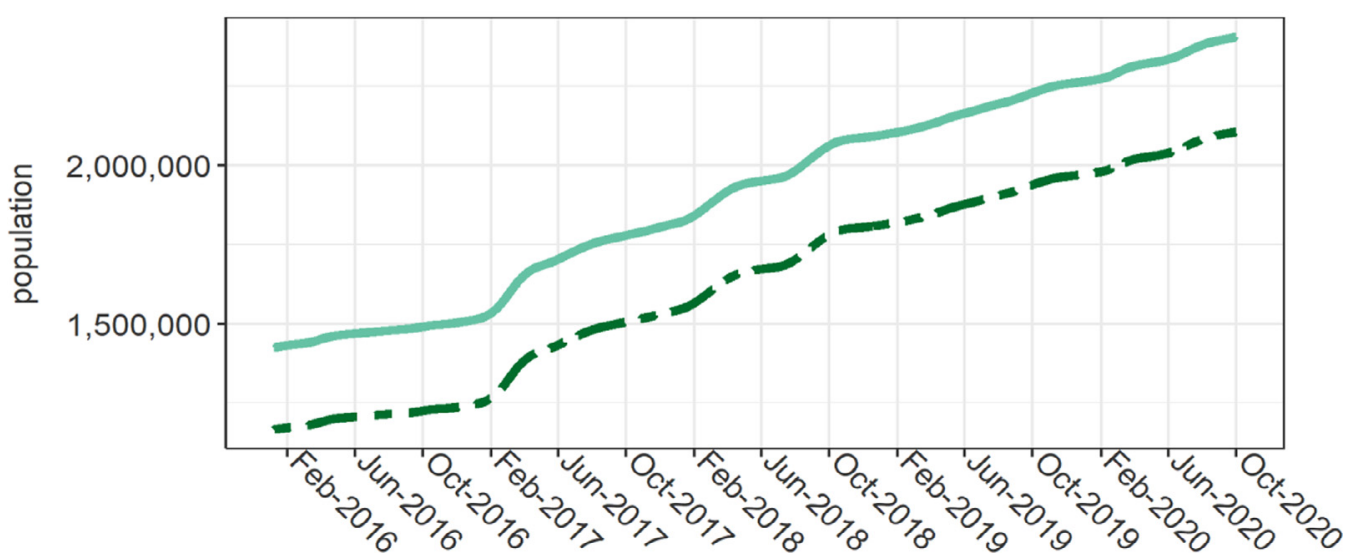

Figure 2. High and low estimates of the population over time in Banadir Region.

tion of, or the totality of, the cemetery, leaving the overall surface area of the cemetery as the sole robustly quantifiable variable. For another subset of images poor image quality precluded a comprehensive grave count. Overall, we were able to perform an exhaustive grave count for $58.8 \%$ (40/68) of images. For eight images (11.7\%), we analysed only the visible area or a sample of $10 \mathrm{~m}^{2}$ quadrats selected randomly by overlaying a grid onto the cemetery. Here, we extrapolated the ratio of graves per $\mathrm{m}^{2}$ observed within the sampled quadrats to approximate the total number of graves (for the first observation in a cemetery) or new graves since the previous image (for further time points). Since graves must increase with expanding cemetery surface areas, we imputed the number of graves in the remaining $20(29.4 \%)$ images by fitting a generalised additive mixed growth model for location, size and shape (GAMLSS) of the count of new graves, with the natural log of new surface area as growth predictor, new surface area nested within the cemetery as a random effect and assuming a quasiPoisson distribution. We then fitted the model with the gamlss package in $\mathrm{R}$ and used it to predict the missing grave observations. Growth models are suitable for processes where a given quantity (in this case, number of graves) increases or decreases as a function of an accumulating variable (in this case, cemetery surface area; in other applications, age or time). Additive models allow for growth to vary non-linearly by estimating smoothing parameters that, taken together, specify a best-fit line through the data. In the GAMLSS framework, the local slope of the growth function can be estimated, and the shape and dispersion of the distribution of new graves (Besson et al., 2021). Lastly, the mixed model allows for specifying random effects that capture variability from one cemetery to the next due to unmeasured characteristics, thus improving model fit. We used the model to predict the missing grave observations. Output from the model is shown in the supplementary file (Table S1), and all data and R scripts are available on Github (https://github.com/francescochecchi/mogadishu_burial_analysis).

Estimating excess mortality. After imputation, we were left with a time series of observed or imputed grave counts for each cemetery. We linearly interpolated each cemetery time series (i.e., connected each observation with a straight line) to obtain daily values and summed each daily time series to compute an overall trend for all cemeteries analysed, i.e., our estimate of burials across the Banadir region. We bound estimation to the period for which data were available for all cemeteries (1 January 2017 to 16 September 2020). We computed daily burial rates per 10000 person-days for each alternative population estimate. After inspecting the re- sulting trend, we decided that the most reasonable (albeit crude) approach for defining a pre-COVID-19 baseline level of burials was to fit a smooth spline function to the trend in burials pre-2020 and extrapolate this function forward into 2020 to compute a nonpandemic counterfactual.

Since the burial rate estimated from satellite imagery appeared implausibly low, we used an indirect method for excess mortality estimation, informed by a range of plausible pre-pandemic CDR values. Specifically, we scaled the estimated burial rate at 1 January 2017 to CDR values within the range of 0.20 to 0.60 per 10 000 person-days: this reflects the range of results of rapid anthropometric and retrospective mortality surveys done by humanitarian actors between 2013 and 2018 in different communities of Banadir Region (Table 1 ). These surveys were conducted utilizing a standardized and validated methodology among populations with different livelihoods in both the major (Deyr) and minor (Gu) rainy seasons (FSNAU, 2011).

We used each scaled time series of baseline CDR, combined with the observed daily ratios of estimated to counterfactual burial rate during 2020 and population denominators, to estimate the actual and excess death rates across the Banadir Region, and the corresponding death tolls.

\section{Qualitative data analysis}

We took a thematic analysis approach toward the key informant data (Bryman, 2012). Key informant interviews were conducted in Somali and transcribed from audio files to text. Transcripts were proofread, cleaned and then translated into English for coding and analysis. Initial coding was performed by two team members and reviewed for fit by a further two team members. Upon agreement, the codes were applied to all the transcripts. Themes were developed after the categorisation of codes. The analysis of the qualitative interviews was triangulated with other data sources of the study.

\section{Results}

\section{Availability of satellite imagery}

We included six cemeteries in our analysis across Mogadishu and Banadir for which we were able to source imagery that met the inclusion criteria (Table 2). The number of images varied among sites from eight to 14 . The earliest images were from January 2016 and the latest from October 2020. In total, we tallied 18 616 burials across all sites during this period. 
Table 2

Availability of imagery and characteristics of cemeteries included in the analysis.

\begin{tabular}{|c|c|c|c|c|c|c|c|c|c|c|}
\hline \multirow{2}{*}{ Cemetery } & \multirow{2}{*}{ District } & \multirow{2}{*}{ Typology } & \multicolumn{2}{|c|}{ Status } & \multirow{2}{*}{$\mathrm{N}$ images } & \multicolumn{2}{|c|}{ Imagery timespan } & \multirow{2}{*}{$\begin{array}{l}\text { New graves } \\
\text { during the } \\
\text { period }\end{array}$} & \multicolumn{2}{|c|}{ Surface area $\left(\mathrm{m}^{2}\right)$} \\
\hline & & & 2016 & 2020 & & Earliest & Latest & & $\overline{\text { Start }}$ & End \\
\hline Barakaat 1 & Yaqshid & $\begin{array}{l}\text { New blocks } \\
\text { and infilling }\end{array}$ & existed & closed & 14 & $20 / 02 / 2016$ & $01 / 10 / 2020$ & 5562 & 10,674 & 95,856 \\
\hline Barakaat 2 & Heliwaa & $\begin{array}{l}\text { New blocks } \\
\text { and infilling }\end{array}$ & did not exist & open & 9 & $17 / 11 / 2019$ & $25 / 09 / 2020$ & 1992 & 0 & 18,391 \\
\hline Calamada & Afgooye & New blocks & existed & open & 11 & 19/01/2016 & $16 / 09 / 2020$ & 5161 & 15,574 & 41,155 \\
\hline Iskool Bolisii & Xamarjajab & New blocks & existed & open & 8 & $02 / 11 / 2016$ & $25 / 09 / 2020$ & 720 & $\mathrm{n} / \mathrm{a}$ & $\mathrm{n} / \mathrm{a}$ \\
\hline Kahda & Kaxda & Infilling & existed & open & 13 & $03 / 10 / 2016$ & $01 / 10 / 2020$ & 1325 & 5012 & 12,190 \\
\hline Moallim Nuur & Deynile (Garasbaley) & Infilling & existed & open & 12 & $07 / 01 / 2016$ & $25 / 09 / 2020$ & 3856 & 72,335 & 93,442 \\
\hline
\end{tabular}
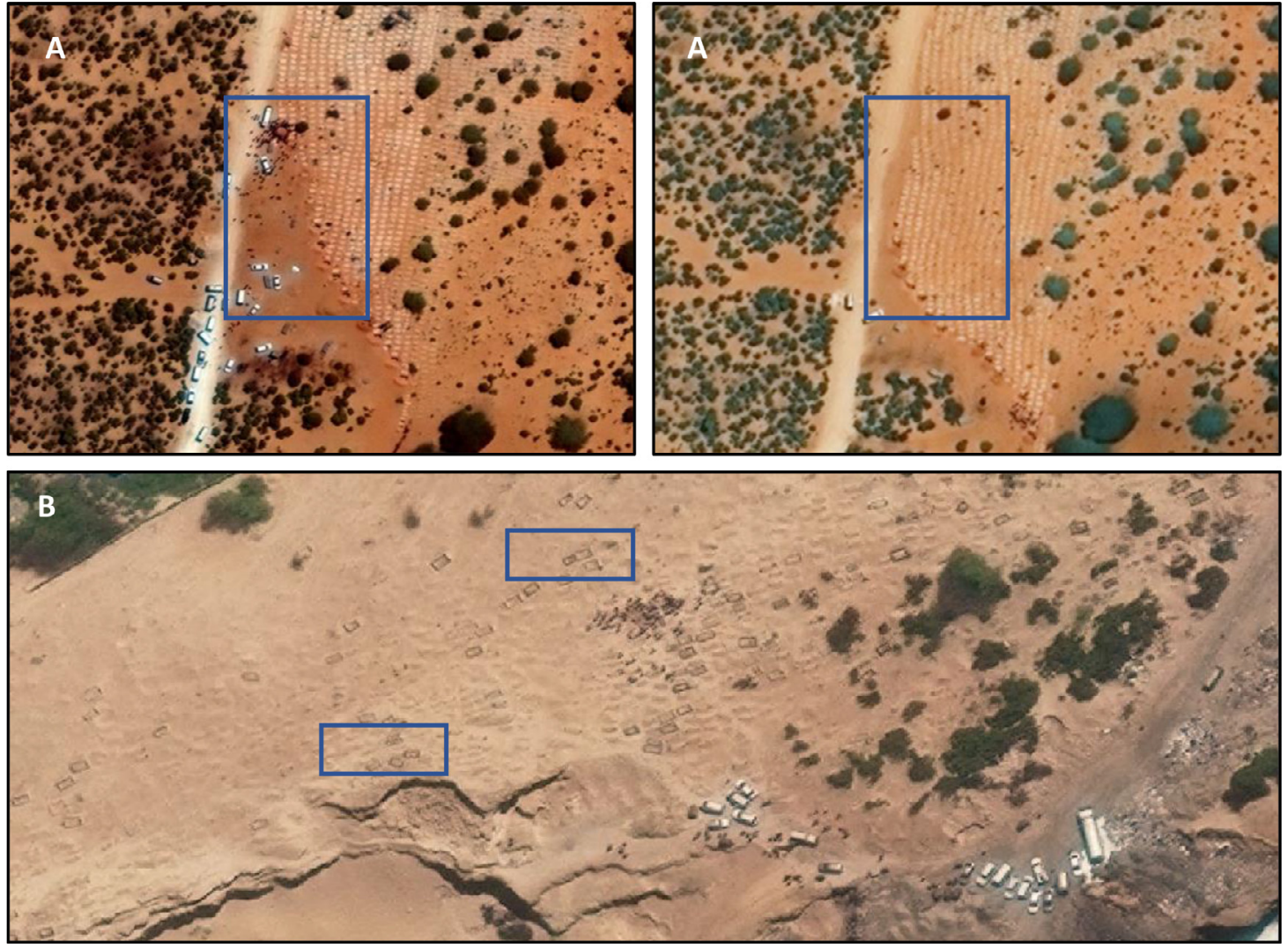

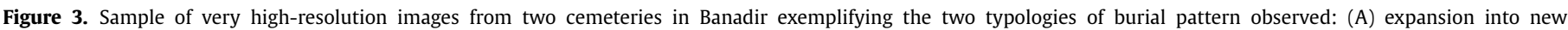
'blocks'(June-September 2020), and (B) 'infilling' (June 2020) within existing burial area Satellite imagery (C) Maxar Technologies 2021.

Our analysis shows that cemeteries varied greatly in size. The largest, Barakaat One, had an estimated surface area of 95856 $\mathrm{m}^{2}$ and the smallest, Kahda, was $12190 \mathrm{~m}^{2}$ by the end of the study period. In general, the largest burial sites were located in less densely populated areas, in many cases on the outskirts of Mogadishu.

Cemeteries also displayed a marked difference in expansion patterns. In the larger, professionally managed cemeteries, new burials occurred in new evenly spaced blocks at the edges of the cemetery, thereby expanding the overall area of the cemetery. Other sites, presumably due to limitations in space, utilized 'infilling' with new burials observed between existing burial rows (Figure 3).

Burial rates across the cemeteries demonstrated variability in the mean new graves observed per day during the analysis period. Some cemeteries displayed a relative increase in burials during the epidemic period (January 2020 onwards), with Iskool Bolisi and Barakaat Two showing the most marked increase relative to baseline.

\section{Burial trends and excess mortality}

Interpolated trends in daily burials by cemetery are shown in Figure 5. During the baseline period (2017-2019), daily burials across the six cemeteries averaged $\sim 10-12$ (panel A). From January 2020 , an increase in burials, peaking at $>20$ daily in June 2020, was evident. Note that trends in 2017-2019 are informed by only a few images (panel B) and may mask a more unstable pattern than that derived through our interpolation (i.e., unseen peaks and troughs).

When combined with alternative population denominators, the above estimates yielded daily burial rates per 10000 population of $\sim 0.06-0.08$ in January 2017, depending on the population source, declining to $\sim 0.04-0.05$ by the end of 2019 (Figure 6). Burial rates increased during 2020 (Figure 7) with a ratio to pre-pandemic levels averaging 1.5 -fold and peaking at 2.2-fold.

Even before the pandemic, our estimated burial rates were approximately one-third to one-tenth of the range in CDR estimated by previous surveys in Banadir (Table 1 ). When scaled to this plau- 

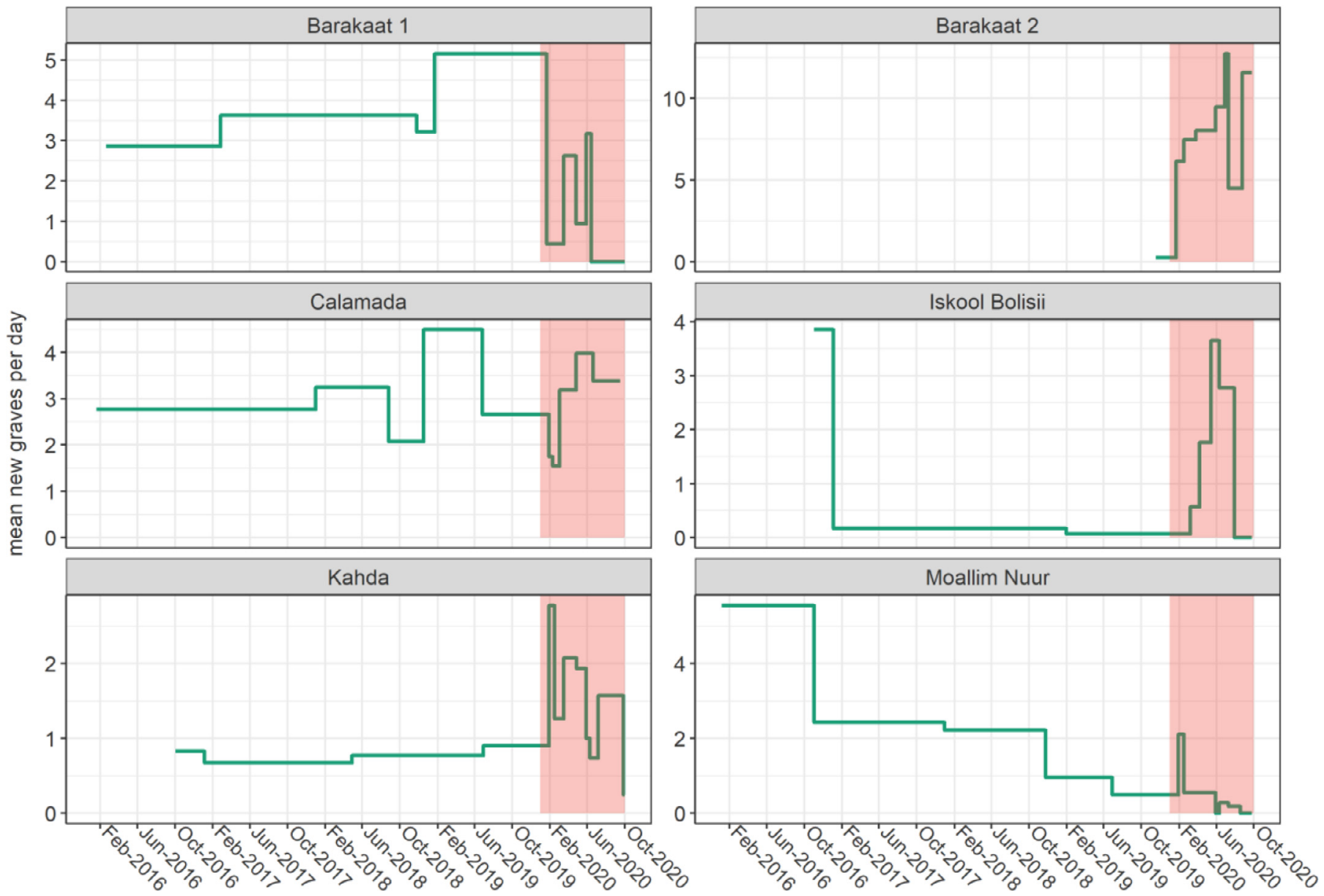

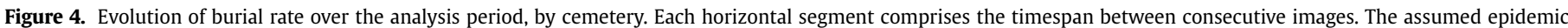
period (1 January 2020 onwards) is shaded in red. Note that the y-axis scales are cemetery-specific.

Table 4

Comparison of alternative burial sources, for months with sufficient data.

\begin{tabular}{lllll}
\hline Cemetery & Year & Month & Satellite imagery & Barakaat Development Committee \\
\hline Barakaat $1+2$ & 2019 & 4 & 155 & 494 \\
Barakaat $1+2$ & 2019 & 5 & 160 & 532 \\
Barakaat $1+2$ & 2019 & 6 & 155 & 473 \\
Barakaat $1+2$ & 2020 & 1 & 178 & 778 \\
Barakaat $1+2$ & 2020 & 2 & 209 & 389 \\
Barakaat 1 +2 & 2020 & 3 & 279 & 390 \\
Barakaat 1 +2 & 2020 & 4 & 313 & 609 \\
\hline
\end{tabular}

sible range of baseline CDR, the excess death toll between January and September 2020 ranges between 3200 and 11800 depending on the assumptions used (Table 3 ).

\section{Comparison with other available sources}

When compared with figures provided by Barakaat Cemetery Development Committee, our estimates were substantially lower.

\section{Key informant interviews}

Informants stated that government-imposed COVID-19 healthrelated measures were not effectively observed. In March 2020, authorities declared a lockdown and imposed restrictions on public movements and meetings, including the closure of schools, government offices and restriction of international travel. In reality, community interactions and public gatherings continued to func- tion normally as hotels, teashops, mosques, and other public places remained open.

Informants reported an increase in the number of deaths in the month of Ramadan (April-May). A few respondents reported some of these deaths as being caused by a flu-like disease. Informants also identified a capacity gap among health care professionals and inadequacy of health equipment such as ventilators, personal protective equipment, and other rudimentary equipment. "Shortage of ICU beds contributed to deaths as a majority of the dead died of breathing difficulties. Imagine less than 20 ICU beds are available in Mogadishu where almost 4 million persons live" (local health professional, November 2020). Informants also mentioned elevated prices of face masks and antiseptics as a barrier to access for the population, potentially hampering the containment of COVID-19.

The responses from the key informants appear to point towards an overburdened public health system struggling to deal with the increasing severity of the epidemic, which is consistent 
A

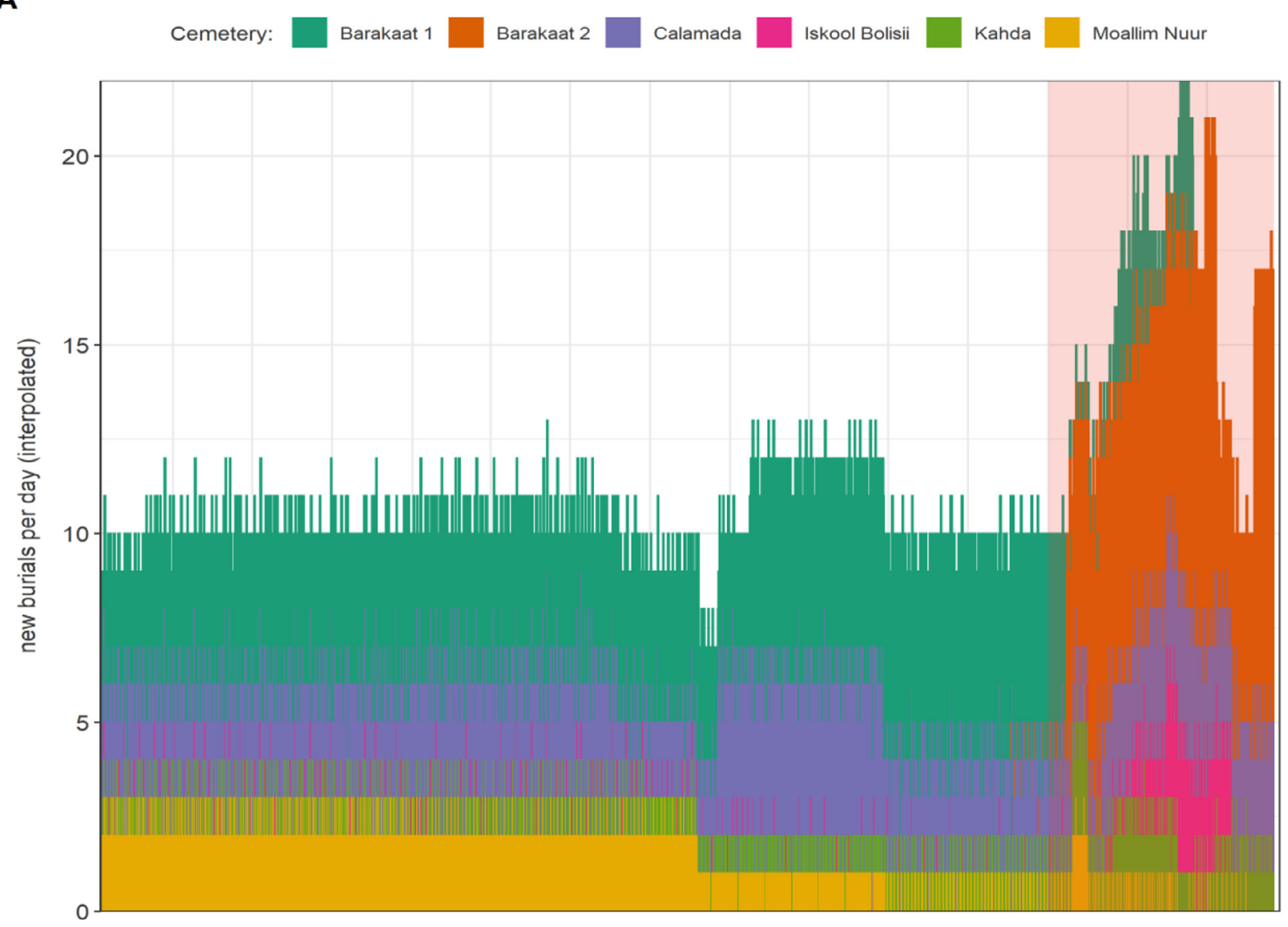

B

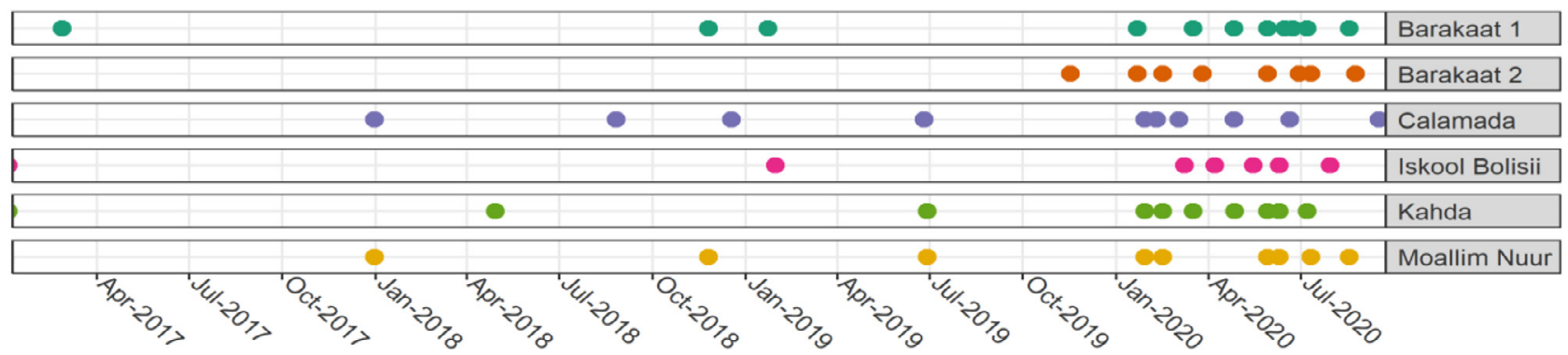

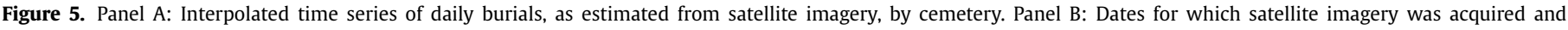
analysed, by cemetery.

with the evolution in burial activity over the analysis period shown in Figure 5, especially at Barakaat One and Two cemeteries. "In May this year, we have seen a substantial increase in the number of deaths almost we have seen 600 people in one month. We've never seen such increase in the number of deaths" (BCDC member, November 2020). Informants also highlighted the stigma surrounding COVID-19 and its role in driving people to seek care at home rather than in hospitals. "Families refused to take their ailing patients to the de Martini hospital fearing that they will not be able to see them again once admitted" (local health professional, November 2020). Lastly, respondents reported a lack of appropriate COVID-19 burial protocols at funerals. Somalis predominantly follow traditional Islamic burial practices. Upon death, the decedent is ritually washed by individuals of the same gender. A congregational funeral, led by an imam, typically occurs in the mosque and involves both men and women. The decedent is buried by men while women participate as onlookers. The burial occurs on the same date in which death occurs in a simply marked grave. Cre- mation and grave sharing are not practised within Somali culture. These customs have not changed during the COVID-19 pandemic and continue to be practised.

\section{Discussion}

Given the well-characterized delay from infection to death (Baud et al., 2020), the peak of COVID-19 deaths reported by this study suggests a much earlier introduction into Somalia than hitherto believed, possibly as far back as December 2019. During this peak, anecdotal reports from media and government authorities suggest a substantial increase in burial rates (BBC News, 2020; Jason Burke and Abdalle Ahmed Mummin, 2020). Our estimates are broadly in line with initial modelling predications (Projections of COVID-19 epidemics in LMIC countries I CMMID Repository, n.d.). While much of the excess death toll is likely to be COVID-19 cases, some may be attributable to the indirect effects of the pandemic, e.g., socio-economic disruptions or re- 


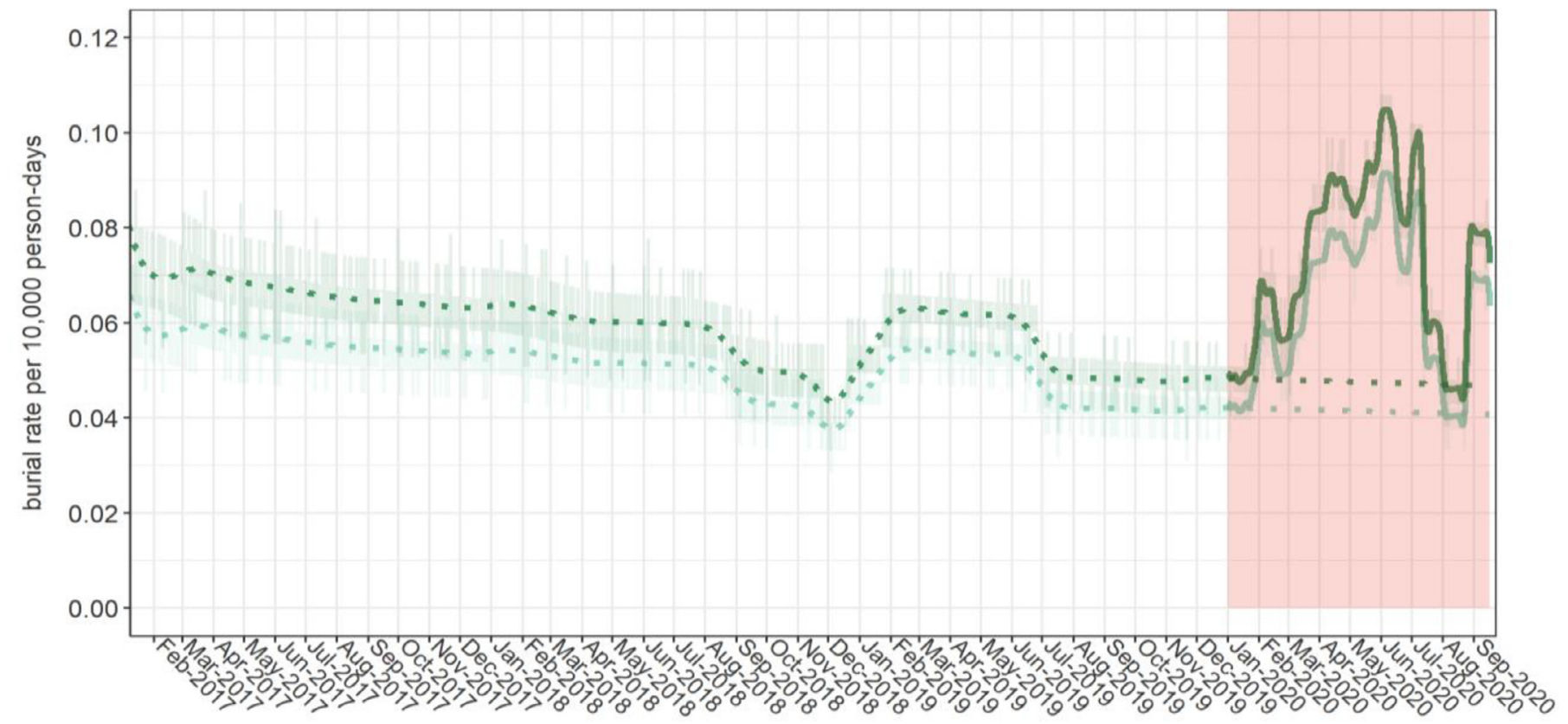

estimate

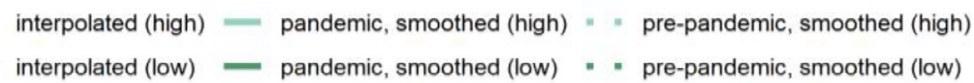

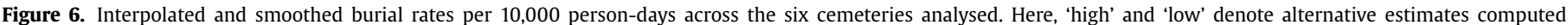

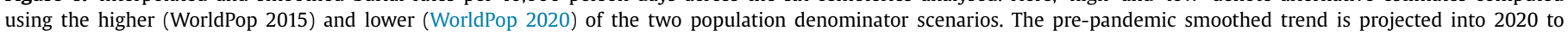
provide a counterfactual level in the absence of COVID-19 and related disruptions.

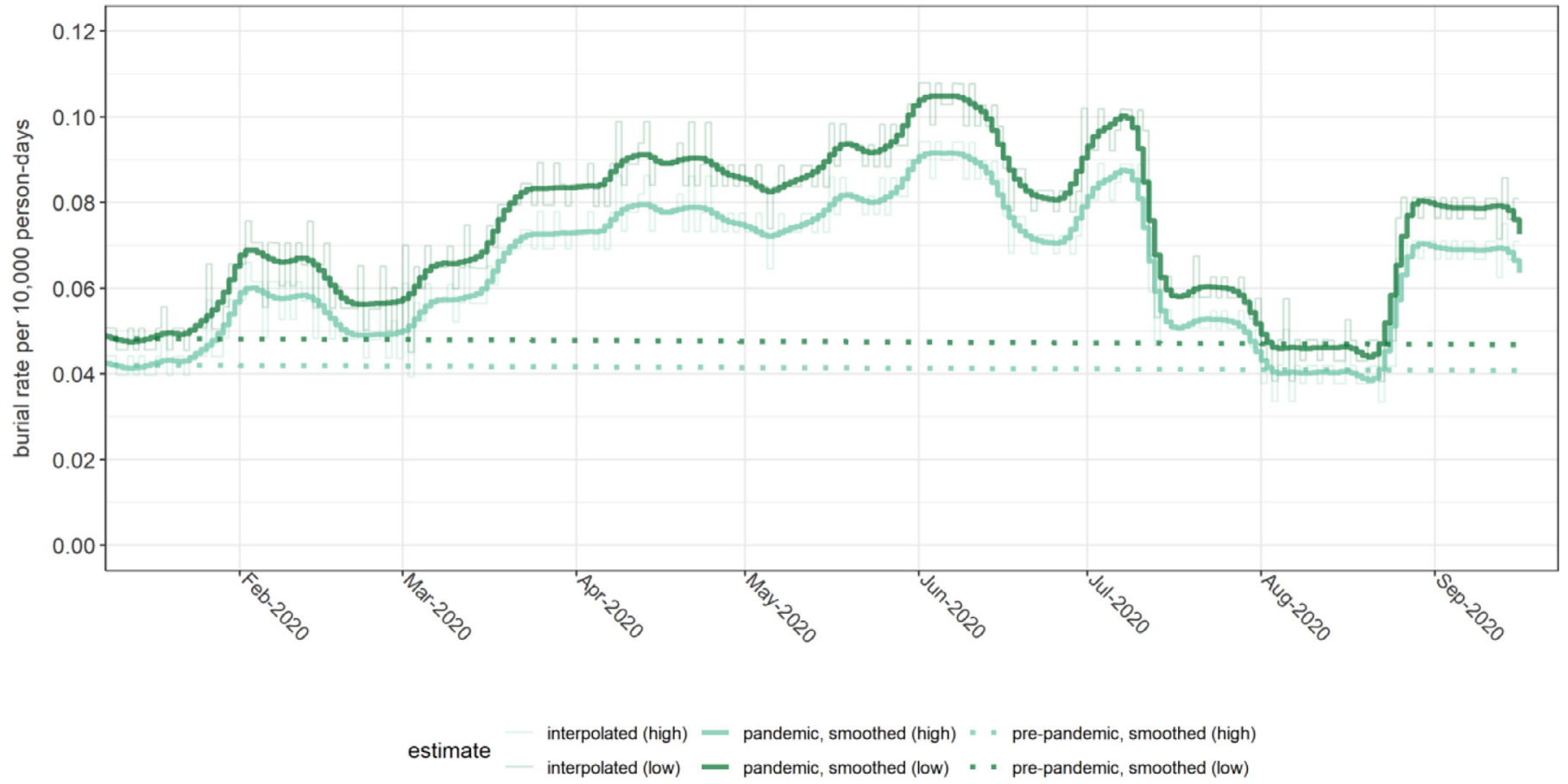

Figure 7. Interpolated and smoothed burial rates per 10,000 person-days across the six cemeteries analysed, during 2020.

duced access to health services due to social distancing restrictions and overwhelmed or repurposed health facilities (Roberton et al., 2020), as has been suggested by research on the COVID-19 pandemic (Giovanni Forchini, Alessandra Lochen, Timothy Hallett, Paul Aylin, Peter J. White, Christl Donnelly, Azra Ghani, Neil Ferguson, 2020) and was documented during the West Africa Ebola epidemic
(Elston et al., 2017). Scepticism about the existence of COVID-19, the lack of social distancing (Shahow, 2021), and the potential for funerals to be superspreader events may have contributed to increased transmission of the virus and could partly explain the scale of excess deaths. Knowledge on preventive measures that may have mitigated the impact of the pandemic is low among the 
Table 3

Estimates of the total, counterfactual (no-pandemic) and excess death tolls in Banadir Region, Jan-Sep 2020, by population estimate, used and assumed baseline crude death rate.

\begin{tabular}{lccc}
\hline $\begin{array}{l}\text { Baseline } \\
\text { CDR }^{\dagger} \text { (as of } \\
1 \text { Jan 2017) }\end{array}$ & Total & Baseline (counterfactual) & Excess \\
\cline { 2 - 3 } 'High' population scenario (WorldPop 2015) & \\
0.20 & 11,200 & 7,300 & 3,900 \\
0.25 & 14,000 & 9,100 & 4,900 \\
0.30 & 16,800 & 10,900 & 5,900 \\
0.35 & 19,600 & 12,700 & 6,900 \\
0.40 & 22,400 & 14,500 & 7,900 \\
0.45 & 25,200 & 16,300 & 8,900 \\
0.50 & 28,000 & 18,100 & 9,900 \\
0.55 & 30,800 & 20,000 & 10,900 \\
0.60 & 33,600 & 21,800 & 11,800 \\
'Low' population & scenario (WorldPop & $2020)$ \\
0.20 & 9,200 & 6,000 & 3,200 \\
0.25 & 11,500 & 7,500 & 4,000 \\
0.30 & 13,800 & 9,000 & 4,900 \\
0.35 & 16,100 & 10,500 & 5,700 \\
0.40 & 18,400 & 12,000 & 6,500 \\
0.45 & 20,800 & 13,500 & 7,300 \\
0.50 & 23,100 & 15,000 & 8,100 \\
0.55 & 25,400 & 16,500 & 8,900 \\
0.60 & 27,700 & 18,000 & 9,700 \\
\hline
\end{tabular}

$\dagger$ Crude death rate per 10,000 person-days.

general population and internally displaced persons in Mogadishu (Alawa et al., 2020). Additionally, fear and stigma associated with hospital care and a preference for homecare (Tanne et al., 2020) could both have increased the proportion of unreported deaths and burials. As with a similar study in Yemen, results indicate a considerable undercounting (Besson et al., 2021) of excess deaths. Given the scarcity of COVID-19 testing in Somalia, such an estimate provides a useful metric for establishing the full health effect of the pandemic.

Ultimately, a long-term COVID-19 control strategy for Somalia needs to be predicated upon sound public health principles. Renewed lockdowns and harmful socio-economic restrictions may not be acceptable, and their health impact is increasingly documented. Instead, community-led, culturally appropriate forms of mitigation may warrant consideration (UNICEF et al., 2021). In particular, local approaches to shield the elderly and vulnerable for a limited period during COVID-19 waves could be adopted by people, with support from humanitarian and development actors (Dahab et al., 2020; van Zandvoort et al., 2020). Social awareness and preventive measures should be prioritized as the weak health system in Somalia cannot provide services to persons with severe cases of COVID-19. Such strategies should be combined with a pragmatic approach to surveillance, utilising not just formal testing but also symptom monitoring (e.g., through social media or sentinel surveillance in health facilities) as well as mortality data collection to rapidly detect and assess the severity of renewed COVID19 waves, whenever these occur.

\section{Limitations}

Systematic or random error may have arisen in our method for imputing missing grave counts. Extrapolation from limited area samples to fill in some of the starting values likely increased error. Moreover, the model to impute missing graves had moderate precision, likely due to the low number of observations used to train it. Further error may have arisen from problems with counting individual graves in satellite images. The above sources of error arise from different statistical processes that are difficult to combine into a single estimation framework. As such, we were unable to produce realistic confidence intervals for the estimates. Over- all, there was substantial evidence that our estimates of burials per population were a considerable underestimate of the plausible death rate in the Banadir region. Our underestimation may have been due to lack of sensitivity in imagery analysis, failure to comprehensively identify and analyse all burial sites used by the population, decedents being buried in their communities of origin outside Banadir region, and/or burials taking place in informal plots (e.g., nearby private residences). Scaling the baseline burial rate to a more reasonable CDR level rests on a strong assumption that the underestimation bias of our method remains approximately constant over time. We undertook satellite imagery analysis retrospectively; therefore, data were limited to what had already been sporadically collected by VHR satellite imagery providers. Additionally, budgetary limitations meant that acquiring new data on demand at $30 \mathrm{~cm}$ for Banadir was impossible, leading to reliance on archive data, thus limiting the number of valid data points we could collect. Undertaking similar studies on a proactive basis would facilitate the collection of VHR data promptly at a cadence which would allow for a greater density of data points both in space and time. The high cost and competition for satellite data is a limiting factor of this approach; however, this can be addressed through increased funding for data coupled with preferential agreements with satellite data providers. Lastly, the detection algorithm developed cannot detect graves in areas where the ground has degraded due to erosion or where tree growth has obscured the grave plots. Additional validation fieldwork would significantly improve burial estimates; however, due to the sensitive nature of the research and limited access to field sites, this was not possible in this study. Future research could benefit from these approaches. The expected availability of an increased number of satellites with higher resolution, increased temporal revisit, and lower-cost data that could be negotiated in the coming years will allow for more precise burial counts, less reliance on interpolation, and potentially broader applicability in similar settings.

\section{Conclusions}

The findings from this study suggest substantial underreporting of COVID-19 in Mogadishu, Somalia. As with our previous work in Aden governorate, Yemen, we believe the satellite imagery method coupled with local verification and qualitative data is a promising epidemiological tool for assessing excess mortality in resource-constrained and fragile/crisis-affected settings. Our study suggests that the Somali population is highly vulnerable to COVID19 , with no evidence of reduced susceptibility after accounting for age differences between Somalia and high-income countries. The current observation of a severe renewed wave suggests that existing immunity was insufficient to grant herd protection, either due to waning of immunity or the introduction of more transmissible variants. These observations underscore the importance of reinforcing control measures for COVID-19, including behaviour change and hygiene, and rolling out COVID-19 vaccination, at least to highly vulnerable groups within Somalia Figure 4 Table 4

\section{Conflict of Interest}

The authors declare no conflict of interest.

\section{Funding}

The study was funded by the United Kingdom Foreign Commonwealth and Development Office (FCDO) through separate grants to the Somali Disaster Resilience Institute and the Satellite Applications Catapult, Inc. AW and FC were supported by UK Research and Innovation as part of the Global Challenges Research Fund, grant number ES/P010873/. 


\section{Ethical approval}

Ethical approval was received from the ethics review committee of the London School of Hygiene and Tropical Medicine (REF: 22458) as well as the ethics review committee of the Somali Disaster Resilience Institute (REF: RB-0123)

\section{Acknowledgements}

We would like to acknowledge Sheikh Abdullahi Ali of the Barakaat Cemetery Development Committee, Dr Khalif Bile of the Somali Swedish Researchers' Association and Dr Mohamed Fuje of the national COVID-19 task force for their support.

\section{Supplementary materials}

Supplementary material associated with this article can be found, in the online version, at doi:10.1016/j.ijid.2021.09.049.

\section{References}

Alawa J, Al-Ali S, Walz LA, Wiles E, Harle N, Awale MA, et al. Knowledge and perception of COVID-19, prevalence of pre-existing conditions, and access to essential resources and health services in Somali IDP camps. MedRxiv 2020 2020.08.17.20176271. doi:10.1101/2020.08.17.20176271.

Baud D, Qi X, Nielsen-Saines K, Musso D, Pomar L, Favre G. Real estimates of mortality following COVID-19 infection. Lancet Infect Dis 2020;20:773. doi:10.1016/S1473-3099(20)30195-X.

BBC News. The gravedigger's truth: Hidden coronavirus deaths. Br Broadcast Corp News Africa 2020.

Besson EK, Norris A, Bin Ghouth AS, Freemantle T, Alhaffar M, Vazquez Y, et al. Excess mortality during the COVID-19 pandemic in Aden governorate. Yemen: LSHTM; 2020 A geospatial and statistical analysis.

Besson ESK, Norris A, Ghouth ASB, Freemantle T, Alhaffar M, Vazquez Y, et al. Excess mortality during the COVID-19 pandemic: A geospatial and statistical analysis in Aden governorate, Yemen. BMJ Glob Heal 2021;6:4564. doi:10.1136/bmjgh-2020-004564.

Bryman A. Social Research Methods. Oxford; 2012.

Centre national d'études spatiales. Orfeo ToolBox - Orfeo ToolBox is not a black box 2019. https://www.orfeo-toolbox.org/ (accessed May 6, 2021).

Chan TF, Vese LA. Active Contours Without Edges. IEEE Trans IMAGE Process 2001:10.

Cloudfactory. Image Annotation for Computer Vision: A Guide to Labeling Visual Data for Your Machine Learning Project 2020.

Dahab M, van Zandvoort K, Flasche S, Warsame A, Ratnayake R, Favas C, et al. COVID-19 control in low-income settings and displaced populations: what can realistically be done? Confl Health 2020;14:1-6. doi:10.1186/s13031-020-00296-8.

Directorate of National Statistics Federal Government of Somalia. The Somali Health and Demographic Survey 2020. 2020.

Elston JWT, Cartwright C, Ndumbi P, Wright J. The health impact of the 2014-15 Ebola outbreak. Public Health 2017;143:60-70. doi:10.1016/j.puhe.2016.10.020.
FSNAU. Guidelines for Emergency Nutrition \& Mortality Surveys in Somalia; 2011 Nairobi.

Giovanni Forchini, Alessandra Lochen, Timothy Hallett, Paul Aylin, Peter J. White, Christl Donnelly, Azra Ghani, Neil Ferguson KH. Report 28 - Excess non-COVID19 deaths in England and Wales between 29th February and 5th June 2020. Imp Coll London 2020

Google Earth. 2021. https://www.google.co.uk/intl/en_uk/earth/ (accessed May 6, 2021).

Google Maps. 2021. https://www.google.co.uk/maps/about/\#!/ (accessed May 6, 2021).

Burke Jason, Abdalle Ahmed Mummin. Somali medics report rapid rise in deaths as Covid-19 fears grow. Guard 2020.

Linard C, Gilbert M, W Snow R, Abdisalan M, Noor AJT. Population Distribution, Settlement Patterns and Accessibility across Africa in 2010. PLoS One 2012

Maxar. Maxar-Securewatch 2021.

Ministry of Health and Human Services FG of S. COVID-19 Dashboard, Somalia 2020. http://moh.gov.so/en/covid19/ (Accessed June 22, 2021).

OpenStreetMap. 2021. https://www.openstreetmap.org/\#map=6/54.910/-3.432 (accessed May 6, 2021).

Projections of COVID-19 epidemics in LMIC countries | CMMID Repository. 2021 n.d.

Roberton T, Carter ED, Chou VB, Stegmuller AR, Jackson BD, Tam Y, et al. Early estimates of the indirect effects of the COVID-19 pandemic on maternal and child mortality in low-income and middle-income countries: a modelling study. Lancet Glob Heal 2020;8:e901 -8. doi:10.1016/S2214-109X(20)30229-1.

Shahow AA. The New Humanitarian | Who's afraid of COVID-19? Somalia's battle with the virus. New Humanit 2021 https://www.thenewhumanitarian. org/analysis/2021/5/5/whos-afraid-of-covid-19-somalias-battle-with-virus. (accessed May 11, 2021).

Tanne JH, Hayasaki E, Zastrow M, Pulla P, Smith P, Rada AG. Covid-19: How doctors and healthcare systems are tackling coronavirus worldwide. BMJ 2020;368. doi:10.1136/bmj.m1090.

UNDP. Floods, locusts and COVID-19; Somalia's triple threat - Somalia. United Nations Dev Program; 2020.

UNFPA Somalia. Saving the lives of Somali mothers and newborns amid COVID-19. United Nations Popul Fund; 2020.

UNHCR. SOMALIA: INTERNAL DISPLACEMENTS. 2021 n.d. https://unhcr.github. io/dataviz-somalia-prmn/index.html\#reason=\&month=\&need=\&pregion= \&pdistrictmap=\&cregion=\&cdistrictmap=\&year=2021 (accessed March 30, 2021).

UNICEF, UNFPA, WHO, SickKids' Center for Global Child Health. Direct and indirect effects of the COVID-19 pandemic and response in South Asia; 2021 Kathmandu, Nepal.

UNSC. COVID-19, Severe Locust Outbreaks Compound Economic, Security Woes in Somalia Ahead of Long-Awaited Elections, Experts Tells Security Council | Meetings Coverage and Press Releases. United Nations Secur Counc 2020.

Wariyaha Muqdisho. Filish: Dad badan oo aan garanayo ayaa Covid-19 ugu dhintay Muqdisho. Garowe Online 2020. https://www.garoweonline.com/en/ news/somalia/filish-dad-badan-oo-aan-garanayo-ayaa-covid-19-ugu-dhintaymuqdisho (accessed October 3, 2020).

WHO. Responding to COVID-19 in Somalia : Progress Report 6 months of resilience and strength; 2020.

Wordpop Gridded Population Estimate Datasets and Tools. 2020.

van Zandvoort $\mathrm{K}$, Jarvis $\mathrm{CI}$, Pearson $\mathrm{CAB}$, Davies NG, Nightingale ES, Munday JD, et al. Response strategies for COVID-19 epidemics in African settings: a mathematical modelling study. BMC Med 2020;18:324. doi:10.1186/s12916-020-01789-2. 